\title{
Neurocase
}

\author{
HOME HELP FEEDBACK SUBSCRIPTIONS ARCHIVE SEARCH TABLE OF CONTENTS
}

Institution: Bib Charcot Sev de Neurologie \| $\underline{\text { Sign In as Personal Subscriber }}$

Neurocase (1995) Vol. 1, pp. 121-138

(C) Oxford University Press 1995

\section{Number Processing in Pure Alexia: The Effect of Hemispheric Asymmetries and Task Demands}

\section{Cohen ${ }^{1, \underline{2}}$ and S. Dehaene $\mathrm{e}^{\underline{2}}$}

Abstract of this Article

Similar articles found in: Neurocase Online

This Article has been cited by: other online articles

Search Medline for articles by: Cohen, L. II Dehaene, S.

Alert me when: new articles cite this article

Service de Neurologie, Clinique Paul Castaigne, Hôpital de la Salpêtrière, 47 Boulevard de l'Hôpital, 75651 Paris Cedex 13 and Laboratoire de Sciences Cognitives et Psycholinguistique EHESS, INSERM and CNRS, Paris, France

Correspondence to: L. Cohen.

Top

- Abstract

Introduction

Case descriptions

Language assessment

Processing of arabic numerals

General discussion

REFERENCES

\section{Abstract}

The relative sparing of arabic numerals, in patients who fail to read words or even letters, is a classical feature of pure alexia originally observed by Dejerine (Comptes Rendus des Seances de la Societé de la Biologie 1892; 4: 61-90). We report a study of number processing abilities in two patients suffering from typical pure alexia. Our main finding was that number identification performance varied considerably with task demands. Both patients could name pairs of digits, when they were engaged in a simple naming task or for the purpose of magnitude comparison. In contrast, they frequently misidentified the very same digits when treating them as the components of multidigit numerals, or as the operands of addition problems. With two-digit numerals, a similar dissociation was shown between excellent comparison and severely impaired reading aloud. Finally, the variation of performance with task demands was shown not to prevail with spelled-out numerals. These findings confirm that 
some patients with pure alexia are able to process up to a semantic level symbolic stimuli that they cannot read aloud. We speculate that both hemispheres possess effective digit identification abilities, which are differentially called on depending on the task.

\section{Introduction}

$\underline{\text { Top }}$

$\underline{\text { Abstract }}$

- Introduction

Case descriptions

Language assessment

Processing of arabic numerals

General discussion

REFERENCES

Ever since Dejerine+'s (1892) seminal description of pure alexia, it has often been reported that, in this condition, reading of arabic digits may be relatively spared as compared with reading of alphabetic characters. Although a few tentative explanations have been proposed for this observation (Alajouanine $*$ et al., 1960; Geschwind $\bullet$, 1965a), its empirical value has never been fully appreciated and exploited. In the present study, we revisit the neuropsychological status of arabic numerals in pure alexia. We show that a detailed analysis of the processing of arabic numerals provides strong empirical constraints on both the architecture of number processing models and the understanding of pure alexia.

\section{The canonical account of pure alexia}

Reading aloud a string of characters is a complex task that requires the collaboration of the visual and the linguistic systems. According to standard neuropsychological models of the reading process, the first compulsory step in word processing is the construction, by the visual system, of a representation of the words in a format appropriate for triggering linguistic processes. This representation, which has been termed the 'visual word form' (Warrington* and Shallice, 1980) provides a structural analysis of the visual stimulus into a parsed string of identified characters. The visual word form is hypothesized to provide a common input to all subsequent linguistic processes such as lexical access or spelling-to-sound translation.

This standard model of reading offers an obvious explanation for the word processing deficit of pure alexics. Their visual system would be unable to create an adequate visual word form, thereby impeding all subsequent processing of written material. Lesion evidence is generally consistent with this view. It has long been known that pure alexia results from left posterior cerebral lesions, while similar right-sided lesions leave reading abilities unimpaired (Damasio + and Damasio, 1983). In cases of infarction of the left posterior cerebral artery, the minimal lesion sufficient to yield pure alexia has been tightly localized to the left ventral temporo-occipital region (Binder* and Mohr, 1992). This localization fits well with brain imaging studies of normal subjects (Petersen $*$ et al., 1990; Posner $*$ and Raichle, 1994). 
The precise nature of the underlying visual deficit is still debated and may differ across patients. Available explanations include direct destruction of the visual word form area (Warrington + and Shallice, 1980), visual deafferentation of this area in patients with right hemianopia and an additional callosal lesion (Dejerine + , 1892; Damasio + and Damasio, 1983), or subtle deficits in the processing of complex visual patterns (Farah + and Wallace, 1991; Friedman + and Alexander, 1984). Regardless of the exact neurological cause, however, it is consistently assumed that pure alexia is due to an inability to construct a structural representation of written words.

\section{Some empirical difficulties}

The classical framework outlined above provides no reason to expect any difference in performance between words and non-words, between words of various syntactic, lexical or semantic categories, or even between letters and digits. As a matter of fact, however, patients with pure alexia often show some degree of preserved reading abilities. These may include the ability to read single digits and single letters with reasonable proficiency, which is at the basis of the well known letter-by-letter reading strategy. Moreover, a body of recent studies has put to light an unexpectedly wide variety of residual reading abilities. Some patients perform much better in tasks that entail implicit word identification, such as lexical decision or semantic classification, than when attempting to read the same words aloud (for reviews, see Grüsser $\star$ and Landis, 1991; Coslett + et al., 1993). In a letter identification task, some pure alexics may also show a word superiority effect, which implies at least some rudimentary form of lexical access (Bub $*$ et al., 1989; Reuter-Lorenz $*$ and Brunn, 1990).

This body of evidence has led to the suggestion that the word identification abilities of at least some patients with pure alexia may vary with the task or strategy in which they are engaged (Coslett $*$ et al., 1993). Rapid word identification would be minimal when patients are asked to read aloud, but would improve in lexical decision with briefly presented words. We shall argue that the relative sparing of arabic numerals is but an additional instance of those residual, task-dependent, reading abilities, and should be interpreted within a unified framework.

\section{Possible accounts of task effects}

The residual abilities of some pure alexics are obviously incompatible with the classical view of a complete disruption of the visual word form. Two alternative proposals have emerged, which largely parallel the now classical debate on the bases of reading in deep dyslexia (e.g. Coltheart*, 1980; Shallice*, 1988).

The first hypothesis proposes that an incomplete impairment in building up the visual word form could preclude reading aloud while permitting above-chance performance in 'simpler' tasks such as lexical decision or semantic categorization (e.g. Bub + et al., 1989; Farah + and Wallace, 1991; Friedman $*$ et al., 1993). An impaired visual word form might still transmit partial activation to the lexicon. This partial activation may be sufficient to perform better than chance in a lexical decision task. It might also permit the retrieval of some general semantic features necessary for semantic classification tasks. Some reciprocal top-down activation from the lexicon could even account for an advantage of words over non-words in letter recognition tasks. However, this weak lexical activation would not be sufficient to single out the target word from its competitors in the lexicon, a necessary condition for reading it aloud.

An alternative proposal postulates that the residual word identification abilities of pure alexics reflect the operation of a second, independent, word processing system localized in the right 
hemisphere (Shallice + and Saffran, 1986; Coslett $*$ and Saffran, 1989; Grüsser $*$ and Landis, 1991; Coslett $*$ et al., 1993). Several lines of argument have been cited in support of this position. First, studies of split-brain or left hemispherectomized patients (e.g. Gazzaniga $\star$ and Hillyard, 1971; Patterson + et al., 1989) have shown that the right hemisphere may, to some extent, identify visually presented letters and words. Second, the same variables appear to affect the reading performance of pure alexics and of the isolated right hemisphere. The right hemisphere of split brains (Zaidel $\star$ and Peters, 1981) and of left hemispherectomized subjects (Patterson + et al., 1989) is often only capable of processing nouns of high frequency and high imageability, in keeping with some tachistoscopic data obtained in normal subjects (e.g. Hines*, 1977; Eviatar $*$ et al., 1990). The very same variables of grammatical category, frequency and imageability have been shown to influence in a similar fashion the performance of patients with pure alexia (Coslett $\star$ et al., 1993). Third, direct support in favour of the right hemisphere theory was obtained recently by Coslett + and Monsul (1994). They showed that, in a patient with partially recovered pure alexia, oral reading was dramatically disrupted by magnetic stimulation of the right hemisphere whereas left stimulation had no effect whatsoever.

The right-hemispheric framework (see Fig. 1) provides a natural explanation for the influence of task demands on the word identification performance of pure alexics. The intact righthemispheric word identification system would be adequate for tasks that require neither phonological processing nor spoken production, such as lexical decision or semantic categorization. These tasks would be preserved in pure alexia only inasmuch as the words used figure among the limited visual lexicon of the right hemisphere, and also provided that the right hemisphere possesses representations adequate for the required decision (e.g. a representation of coarse semantic categories). However, the right hemispheric word identification system would not be able to provide sufficient inputs to phonological or articulatory processes necessary for reading aloud. This may be because the right visual system does not perform the kind of structural analysis of words that is appropriate to activate phonological structures, or because the callosal connections that could feed the output of a right-hemispheric word identification system to the phonological/articulatory processes of the left hemisphere are absent, insufficiently precise, or have been lesioned.

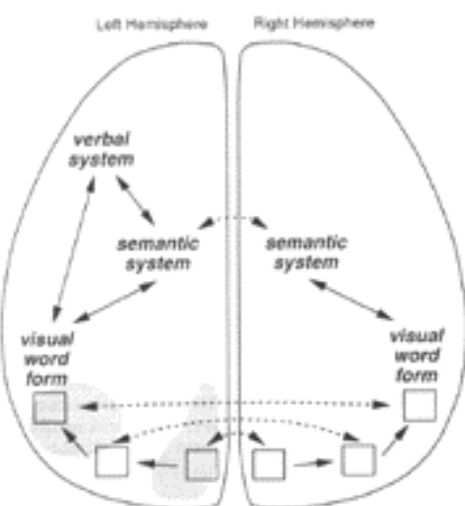

View larger version $(42 \mathrm{~K})$ : [in this window] [in a new window]
Fig. 1 Anatomo-functional model of the reading process incorporating a putative indirect semantic righthemispheric route. Shaded areas represent examples of lesions that should impede the formation of a visual word form and therefore yield pure alexia. 


\section{The case of arabic numerals}

Reading arabic numerals is in many ways similar to reading words. Patients with pure alexia for words generally have difficulties reading multidigit numerals. It seems likely that both deficits reflect the same functional impairment in constructing a structured representation of strings of characters. In the case of numbers, this representation has been termed the visual number form, by analogy with the visual word form (Cohen $*$ and Dehaene, 1991).

As in the case of words, an influence of task structure on number reading has been observed in pure alexia. Thus, in most such patients, error rates per digit are lower when they are reading aloud isolated arabic digits (e.g. 6 $\rightarrow$ 'six') than when they are reading aloud multidigit numerals (e.g 46 $\rightarrow$ 'forty-six') (Dejerine + , 1892; Holender $\star$ and Peereman, 1987; McNeil $\star$ and Warrington, 1994). It should be stressed that despite their apparent similarity, these two reading tasks show deep functional differences. Thus, reading aloud digits only requires the production of the number word corresponding to the stimulus, a task very similar to object naming, at which patients with pure alexia can be quite good.

Reading aloud multidigit numerals, on the other hand, requires the parsing of a string of digits and the construction of a syntactically complex sequence of number words (McCloskey $\bullet$ et $a l ., 1990)$. Hence, as for word reading, two alternative explanations may be proposed for the good reading performance with single digits. It may reflect the operation either of a partially defective left-hemispheric system, or of an alternative right-hemispheric reading route only able to deal with single digits.

In the following study, we explore the numerical abilities of two patients suffering from pure alexia. We show that the visual identification of arabic numerals changes dramatically with the task. In particular, it can be fully preserved in some tasks, a finding which is hard to reconcile with an incomplete impairment of a single identification system. We propose that the results can only be accounted for by appealing to two number identification systems, possibly residing in different hemispheres, and which may be separately called upon depending on the task.

\section{Case descriptions}

$$
\begin{aligned}
& \text { Top } \\
& \text { Abstract } \\
& \text { - Case descriptions } \\
& \text { Language assessment } \\
& \text { Processing of arabic numerals } \\
& \underline{\text { General discussion }} \\
& \underline{\text { REFERENCES }}
\end{aligned}
$$

\section{Patient G.O.D.}




\section{Medical History}

The patient was a 71-year-old right-handed man with a history of arterial hypertension. Before retiring, he had been a radio and television technician. He was admitted to the hospital for atrial fibrillation. Just following cardioversion to a sinus rhythm with amiodarone, the patient presented moderate confusion and right homonymous hemianopia. Confusion receded rapidly, and neurological examination revealed, in addition to the hemianopia, severe alexia without agraphia, some word finding difficulties, and moderate right spatial neglect. There was no sensory or motor deficit. CT scan (see Fig. 2) showed an infarct in the peripheral territory of the left posterior cerebral artery, affecting the calcarine cortex, the lingual and fusiform gyri, and the underlying white matter.

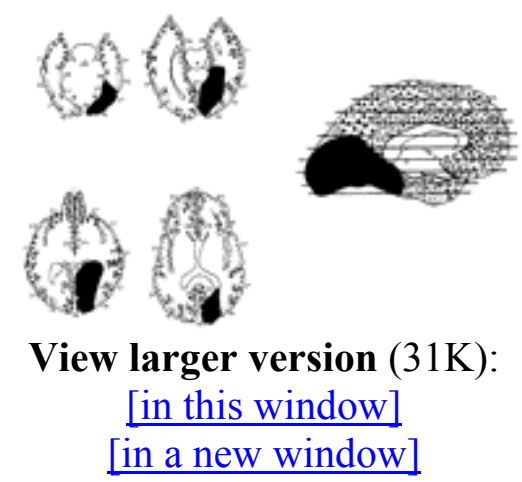

Fig. 2 Mesial view of the left hemisphere and transversal sections of patient G.O.D.'s brain reconstructed on the basis of CT scan, using templates from Damasio + and Damasio (1989). The left hemisphere is represented on the right side of transversal sections.

\section{Neuropsychological evaluation}

The present study was carried out one month after onset. At that time, the patient was alert, oriented and cooperative. Oral speech production and comprehension were normal on clinical examination. Forward digit span was of five items. There was no more evidence of spatial neglect in a line bissection task or in description of complex pictures. In constructional tasks, the patient had some difficulties in accurately placing the parts of a drawing relative to one another. He reported seeing colours quite normally, but showed a mild colour naming defect, a disorder commonly associated with pure alexia (Damasio + and Damasio, 1983). He could easily recognize and name famous faces. There was no visual agnosia for objects. The patient made only two errors in naming a series of 40 line drawings of objects selected from the set in Snodgrass + and Vanderwart (1980), including one visually plausible mistake.

Reading and writing will be described below.

\section{Patient S.M.A.}

\section{Medical history}

The patient was a 77-year-old right-handed retired diplomat. He had a history of diabetes, arterial hypertension and myocardial infarct. One evening, he suddenly presented right hemianopia, and was admitted to the hospital, where alexia without agraphia was diagnosed. Two days later, right-sided numbness and paraesthesias appeared, as well as some degree of confusion and disorientation. Neurological examination showed right-sided hypaesthesia and mild spastic hemiparesis. There was still a dense right homonymous hemianopia without sparing of the macula on Goldmann perimetry. The patient also had marked difficulties in 
reporting the chronological succession of past public events. CT scan (see Fig. 3) disclosed an infarct in the territory of the left posterior cerebral artery, affecting the calcarine cortex as well as the lingual and fusiform gyri. The lesion had a smaller anterior and superior extension than in patient G.O.D. There was another infarct in the posterior part of the left thalamus. These two lesions probably corresponded to the first and second neurological episodes, respectively, the occipital lesion being obviously responsible for the alexia.

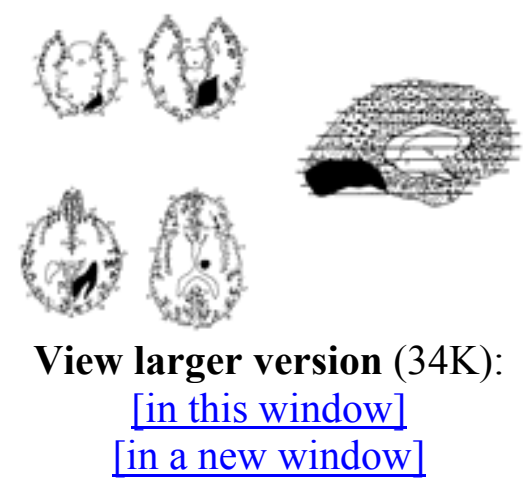

Fig. 3 Reconstruction of patient S.M.A.'s brain on the basis of CT scan.

\section{Neuropsychological evaluation}

The present study was carried out one month after onset. At that time, the patient was alert and cooperative, but not perfectly orientated in time. Oral speech production and comprehension were normal on clinical examination. There was no spatial neglect, and no limb apraxia. All constructional tasks, as well as writing, were notably impeded by clumsiness of the right hand. The patient reported seeing colours quite normally, but showed a mild colour naming defect. He could easily recognize and name famous faces. The patient made four errors in naming the series of 40 line drawings described before. A substantial memory deficit was evidenced on formal testing. The patient had a verbal memory quotient of $76(1.74$ SD below the mean) on the Wechsler Memory Scale Revised, and a total recall of 15 out of 48 $(n>44)$ on the memory test of Grober $*$ et al. (1988).

\section{Language assessment}

$\underline{\text { Top }}$

Abstract

Introduction

Case descriptions

- Language assessment

Processing of arabic numerals

General discussion

REFERENCES 
The patients were submitted to a French version of the Boston Diagnostic Aphasia Examination (Goodglass $\star$ and Kaplan, 1972; Mazaux + and Orgogozo, 1982).

Spontaneous speech was normal, except for mild word finding difficulties in G.O.D. As shown in Table 1, the scores of the two patients were remarkably similar and will be commented on together. They performed at ceiling level in all subtests of fluency, automatic speech, repetition and writing. Owing to S.M.A.'s motor difficulties, a few written words were difficult to decipher, explaining his lower than perfect score on sentence dictation. Both patients also showed a lack of precision in placing words on a line, and occasional reduplications of strokes in such letters as ' $m$ ' or ' $n$ ' were noted. Auditory comprehension was very good, except for the difficult items of the complex ideational material subtest in patient G.O.D. Naming subtests were all correctly achieved. G.O.D.'s errors in confrontation naming included four errors in reading aloud arabic numerals, and one colour naming error, while S.M.A. made one error in each of these two categories. In contrast, both patients were severely and selectively impaired in the reading and reading comprehension subtests. G.O.D. could not correctly read aloud a single item, while S.M.A.'s scores were slightly higher on the word reading and word-picture matching subtests. The patients were rather good at identifying a given target among five written words (word recognition subtest), maybe relying on relatively preserved letter identification abilities (symbol discrimination subtest).

Additionally, the patients were orally presented with a series of 30 nouns three to nine letters long, and asked to write them down (see Table 2). There were no spelling errors, except for occasional duplications of strokes or small letters. The patients were visually presented with the same list of words and asked to read them aloud. Reading was nearly impossible. G.O.D. produced one single correct response ('ville'). He engaged in a series of perseverative responses with the word 'tien' (yours) and testing had to be discontinued before completion of the list. Patient S.M.A. read correctly only two out of 30 words ('cas' and 'moyen'), and produced seven times the word 'quand' (when) as a response. The patients were visually presented with the letters of the alphabet in random order. G.O.D. and S.M.A. both named correctly 15 out of 26 letters, and errors were often perseverative (out of 11 errors, G.O.D. responded ' $\mathrm{K}$ ' four times, and SMA responded ' $\mathrm{H}$ ' six times). A list of 20 words three to seven letters long were spelled out orally to patient S.M.A., who could accurately identify 19 out of 20 items. This task was not proposed to patient G.O.D.

In short, both patients showed typical pure alexia, in the absence of any other significant language disorder. Patient S.M.A.'s additional memory deficit was unrelated to the phenomena studied here and will not be mentioned any more. Both patients had equally typical lesions affecting the left inferomesial occipito-temporal region (Damasio + and Damasio, 1983; Binder $*$ and Mohr, 1992).

\section{Processing of arabic numerals}


As a prerequisite to the experimental study, we first tried to establish whether pure alexia affected arabic numerals in the same manner as it affected alphabetic script. To this end, the patients were asked to read aloud 70 one-digit numerals and 46 two- to four-digit numerals, as well as to write down on dictation 20 two- to four-digit numerals (e.g. 'two-hundred-andthirty-one' was to be written down as 231). Results are summarized in Table 2. $\underline{\text { Top }}$

Abstract

Introduction

Case descriptions

Language assessment

- Processing of arabic numerals

General discussion

REFERENCES

The patients did not make a single error in number dictation. When reading aloud single digits, G.O.D. and S.M.A. made $8.6 \%$ and $18.6 \%$ errors, respectively. As shown in Table 2, error rates were much higher with multidigit numerals (G.O.D.: $80.4 \%$; S.M.A.: $67.4 \%$ ). The patients produced almost exclusively substitution errors, that is, numbers comprising the same number of digits as the target, for instance reading 504 as 'seven hundred and six' (G.O.D.: 35 out of 37 errors [94.6\%]; S.M.A.: 29 out of 31 errors [93.5\%]).

Frequent perseverations were noted. In contrast with this poor performance, patient S.M.A. only made a single error when asked to name 20 complex arabic numerals two to four digits long that were spelled out to him (for instance, 'eight, four, zero' was named as 'eight hundred forty'). Importantly, this latter observation confirms that the patients' reading errors did not result from a faulty production of verbal numerals, but only from an impaired visual identification.

View this table: Table 1. Boston Diagnostic Aphasia Examination [in this window]

[in a new window]

Thus, arabic numerals seemed affected just like alphabetic script. Writing was intact, contrasting with a severe number reading deficit, particularly evident with multidigit numerals. Furthermore, both patients were relatively good at reading single digits aloud, as was previously reported in other pure alexics. Error rates were significantly lower for single digits than for single letters (G.O.D.: $8.6 \%$ versus $42.3 \%, x^{2}(1)=12.58, P=0.00039$, Yates' correction applied; S.M.A.: $18.6 \%$ versus $\left.42.3 \%, x^{2}(1)=5.70, P=0.017\right)$.

Interestingly, there was a striking discrepancy between error rates per digit when the patients read single digits and when they read multidigit numerals (G.O.D.: $8.6 \%$ versus $46.8 \%, x^{2}(1)$ $=29.71, P<10^{-6}$; S.M.A.: $18.6 \%$ versus $34.4 \%, X^{2}(1)=5.47, P=0.019$ ). (The few trials on which the patients' errors could not be considered as digit substitutions were excluded from this analysis.) Whatever process was involved in the identification of single digits, this process obviously became dysfunctional or unavailable for the identification of single digits within multidigit numerals.

\section{Reading aloud pairs of digits}

The variable rate of reading errors per digit in reading aloud complex numerals versus single digits could be explained in two alternative ways. It may reflect differences in task structure, 
such as the fact that reading aloud multidigit numerals, but not single digits, engages complex word assembly processes. Alternatively, it may simply be due to the higher visual complexity of multidigit numerals. In order to elucidate whether the major determinant of reading performance was task structure or stimulus complexity, the patients were presented visually with 20 pairs of two different digits, which were to be read aloud in two different ways.

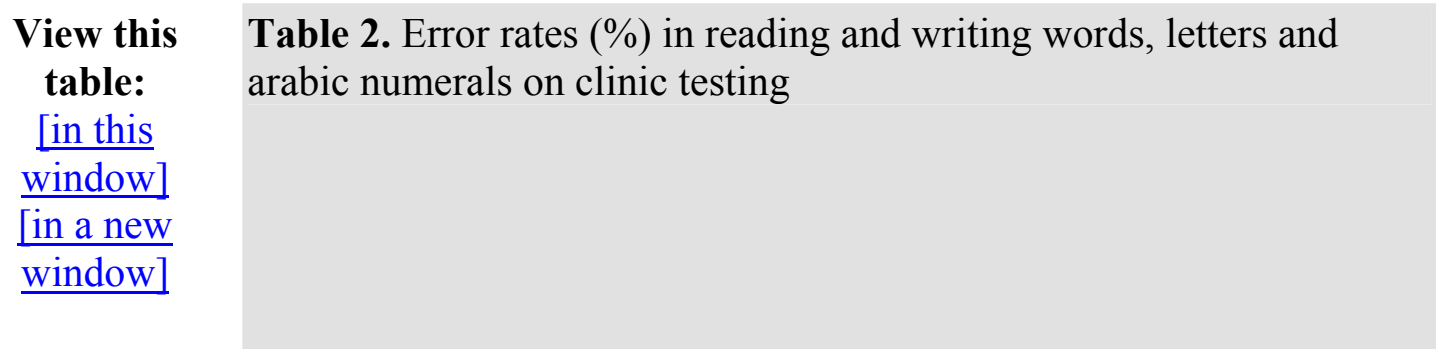

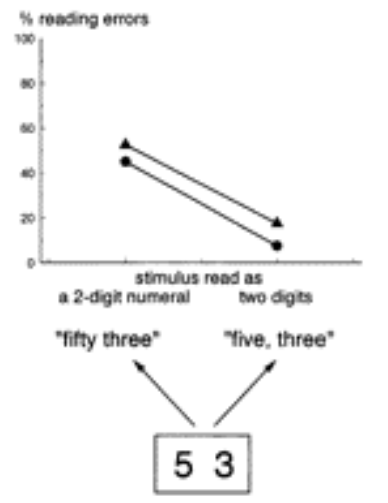

View larger version $(12 \mathrm{~K})$ :

[in this window] [in a new window]
Fig. 4 Errors (per digit) in reading aloud digit pairs as two separate digits versus as a single two-digit number. $\bullet$, Patient G.O.D.; $\Delta[$ solid], patient S.M.A.

On a first presentation of the list (one-digit condition), the patients had to name each digit separately (for example, 24 was to be read 'two, four'). On a second presentation (two-digit condition), they had to name each pair as a single two-digit number (for example, 24 was to be read 'twenty-four'). The pairs of digits were presented horizontally, one at a time, in random order.

The patients only produced names of one-digit numbers in the one-digit condition, and names of two-digit numbers in the two-digit condition. All errors could thus be considered as digit substitutions. As shown in Fig. 4 and Table 3, the response pattern was quite similar in both patients. Error rates per digit were significantly higher in the two-digit condition than in the one-digit condition (G.O.D.: $X^{2}(1)=14.53, P=0.00014$; S.M.A.: $X^{2}(1)=10.77, P=0.0010$ ). 
Furthermore, many perseverations were noted in the two-digit condition only. Hence, it may be concluded that the higher error rate per digit for complex numerals than for single digits resulted from a difference in task demands rather than in stimulus complexity. Indeed, when the task was held constant, the visual complexity of the stimulus had no influence on performance. The patients performed at the same level in the present one-digit condition as when reading single digits on clinical testing (G.O.D.: 7.5\% versus $8.6 \%$ errors; S.M.A.: $17.5 \%$ versus $18.6 \%$ errors).

\section{Digit addition and digit comparison}

We further investigated the influence of task structure on the patient's performance by contrasting digit addition and digit comparison. Those two tasks were chosen because, although they appear superficially similar, a theory of number processing suggests that they involve quite different stages of processing (Dehaene+, 1992; Dehaene + and Cohen, 1995). In the comparison task, arabic numerals must be transformed into a magnitude code, over which the comparison routine is thought to operate (Dehaene $\leftarrow$ et al., 1990). In the addition task, arabic digits must be translated directly into verbal labels, which are then used to retrieve the corresponding result from rote verbal arithmetic memory. In short, the model assumes that addition operates on a verbal representation of numbers, whereas larger-smaller relations are computed from a distinct magnitude code (Dehaene + , 1992; see McCloskey^, 1992, discussed below, for a different model).

We therefore expected that patients with pure alexia, because of their difficulties in reading aloud, might have trouble computing the internal verbal representation required for arithmetic fact retrieval, and hence would perform poorly in an addition task. However, they would have no problem in number comparison, which does not involve the verbal representation.

View this table: Table 3. Dissociations between paired tasks with identical numerical [in this window] stimuli

in a new

window]

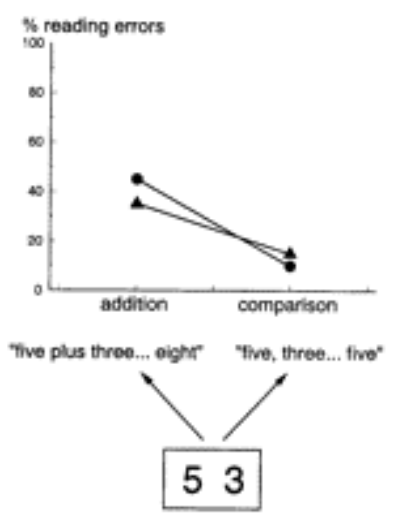

Fig. 6 Errors (per digit) in reading aloud digit pairs for the purpose of adding versus comparing them. •, Patient G.O.D.; $\Delta[$ solid], patient S.M.A.

View larger version 
$(13 \mathrm{~K})$ :

[in this window]

[in a new window]

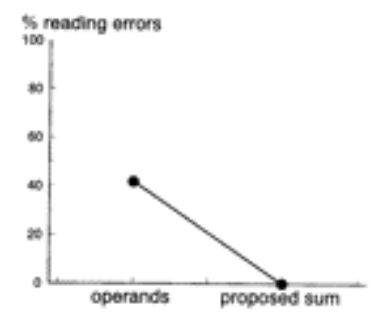

Fig. 7 Errors (per digit) in reading aloud the operands versus the proposed sum of addition problems.

Three plus one... four nine, it's false'

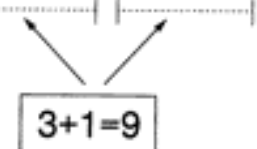

View larger version

$(14 \mathrm{~K})$ :

[in this window]

[in a new window]

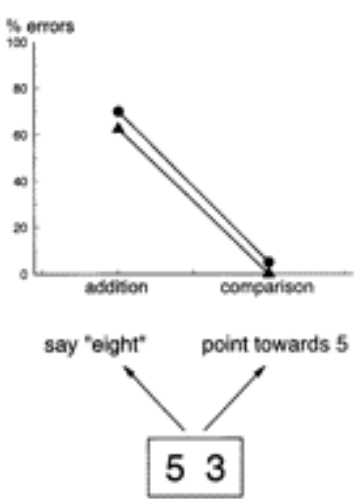

Fig. 5 Errors (per trial) in adding versus comparing digit pairs. • Patient G.O.D.; $\Delta$ [solid], patient S.M.A.

View larger version

$(11 \mathrm{~K})$ :

[in this window]

[in a new window] 


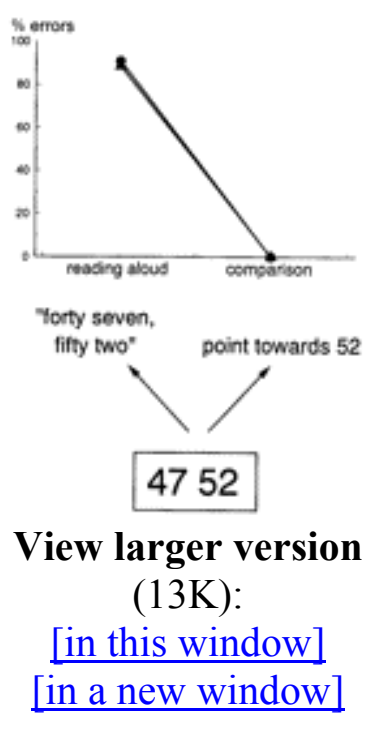

Fig. 8 Errors (per trial) in reading aloud versus comparing two-digit numerals.

Here, the same pairs of digits used previously were presented either for addition or for largersmaller comparison. In the comparison task, patients were asked to point to the larger digit of each pair. In the addition task, they were asked to produce verbally the sum of the two digits of each pair. For instance, the patients were expected to respond 'eight' on presentation of the pair 53 . The 20 pairs were presented once in each condition to patient G.O.D., and twice in each condition to patient S.M.A.

In the comparison task, responses were fast and accurate (see Fig. 5 and Table 3): out of 40 trials, G.O.D. made two errors (5.0\%) and S.M.A. not a single one. The error level was much higher in the addition task: G.O.D. made $70.0 \%$ and S.M.A. $62.5 \%$ errors. Most importantly, on auditory presentation of the same problems, G.O.D. did not make a single error out of 40 addition problems, and S.M.A. made two errors out of 20 addition problems. This contrast between visually and orally presented addition problems is of paramount importance, since it demonstrates that both mental arithmetic and the production of verbal numerals (as already suggested by clinical assessment) were intact. As a consequence, the origin of the errors with visual addition problems should logically be found in the identification of the operands. Therefore we suggest that the dramatic difference in performance level between addition and comparison should be attributed to a difference in digit identification itself.

\section{Digit reading during addition and comparison}

In order to evaluate more directly the hypothesis that digit identification was differentially impaired during addition and comparison tasks, the patients were simply asked to read aloud the operands before performing the two tasks. In the reading-and-comparison task, the patients were asked to read both digits aloud and then to name the larger one and point to it. In the reading-and-addition task, they again named both digits before producing the sum. For instance, on presentation of the pair 53 , the patients were expected to respond 'five, three ... five' in the reading-and-comparison task, and 'five, three ... eight' in the reading-and-addition task. The 20 pairs were presented once in each condition to patient G.O.D., and twice in each condition to patient S.M.A.

Note that in the reading-and-addition task, patient G.O.D. often reordered the operands and named the larger digit first, which is the canonical order in which addition facts are taught at school (reordering was also observed with auditorily presented problems). Patients S.M.A., on 
the other hand, named the digits in their left to right order. For both patients, a conservative estimate of naming errors was computed by considering as correct all responses that could have resulted from a digit inversion. For instance, when presented with the pair 1 7, G.O.D. said 'five and one makes six'. This was scored as a single naming error on digit 7.

In the reading-and-comparison task, neither G.O.D. nor S.M.A. made a single error in pointing to the larger digit (see Table 3 ).

In the reading-and-addition task, they produced an erroneous sum in 15 out of 20 trials $(75.0 \%)$ and in 21 out of 40 trials $(52.5 \%)$ respectively. These error rates were similar to those reported in the previous section, suggesting that reading aloud the operands did not significantly alter the addition and comparison tasks.

More importantly, as shown in Fig. 6 and Table 3, both patients made significantly more errors when reading aloud the very same pairs of digits in the context of addition problems than in the context of comparison problems (G.O.D.: $\chi^{2}(1)=12.29, P=0.00046$; S.M.A.: $X$ $\left.{ }^{2}(1)=5.94, P=0.015\right)$. This demonstrates that the patients were not relying on the same digit identification procedure when solving addition problems and when performing larger-smaller comparisons. Impaired identification, rather than poor calculation, was responsible for the high error rate in the addition task. Indeed, the patients produced the correct sum of the misread digits in all trials but one. For instance when presented with the pair 24 , patient G.O.D. said 'four and six makes ten'. Thus, the addition routine itself was obviously intact.

Patient G.O.D. was also asked to read aloud and solve some multiplication problems using the same digit pairs. His performance was remarkably similar to that observed with addition problems. He produced the wrong result in 11 out of 20 trials $(55 \%)$. All such errors were due to a misreading of one operand (11 errors out of 40 digits [27.5\%]), an error rate higher than in the comparison task $\left(X^{2}(1)=4.02, P=0.045\right)$.

Like in the addition task, he always produced the correct product of the misread digits (for instance, when presented with the pair 49 , he responded 'four times six makes twenty-four'). In contrast, he made only one error out of 20 when the same multiplication problems were presented to him auditorily (he responded 'one' to the problem 'seven times one'), further confirming that both mental arithmetic and verbal output were virtually intact.

In brief, the above results showed that the patients' accuracy in digit identification depended on the task. When engaged in arithmetic calculations, they made more frequent reading errors than when reading single digits or when performing a comparison task.

\section{Addition verification and shift in reading mode}

We then tried to establish how rapidly such reorganization could take place in cases where the two types of processing are mixed within a single task. To this end, we presented patient G.O.D. with an addition verification task. He was required to add two digits and to compare their sum to a proposed result. We expected that, within each trial, the patient would make numerous errors in the addition component, and at the same time perform accurately in the comparison component.

We selected 24 pairs of digits whose sum was also a single digit. Each pair was presented as an addition problem, with a third digit as a proposed sum. The addition was correct in nine problems and incorrect in the other 15 problems. The patient was asked to read aloud the two 
operands, then to compute and produce verbally the sum, and finally to read aloud the proposed sum and to state whether the proposed result was correct or false. For instance, when presented with the problem $3+1=9$, the patient was expected to say 'three and one makes four, nine, it's false'.

Error rates differed widely across the successive stages of the task (see Fig. 7 and Table 3). When reading the operands, patient G.O.D. made $41.7 \%$ errors, a figure similar to the $45.0 \%$ error rate observed in the previous task. As before, he always produced the correct sum of the misread operands. In striking contrast with his poor reading of the operands, he did not make a single error out of 24 digits when reading aloud the proposed result. He was also perfect in deciding whether the proposed result and the result that he had computed himself were identical or different. In summary, the only errors occurred during the identification of the operands and were propagated through all further stages of the task. For instance when presented with $5+4=9$, patient G.O.D. said 'five and three makes eight, nine, it's false'.

These results indicate that patient G.O.D. could rapidly switch between different reading modes and made digit identification errors only in the context of additions but not in the context of same-different judgements.

It seems somewhat paradoxical that the patient appeared to possess, at least in some situations, an adequate digit reading routine, yet was not able to use it at all times. The switching between reading modes, however, appeared to be under partial attentional control. For instance, with the same problems, the patient was instructed to read aloud each of the three digits, disregarding the + and $=$ signs, and then look up and mentally decide whether the operation was correct or not. In this situation, he made only six reading errors out of 72 digits $(8.3 \%)$, none of which entailed any verification errors. Thus it was possible to induce him to read the component digits of addition problems as if they were isolated digits, but this was not his default strategy.

\section{Reading aloud and comparing two-digit numerals}

So far, the influence of task context was demonstrated only with single digits. We now examine if this effect generalizes to multidigit numerals. We have seen that reading aloud multidigit numerals was disproportionately impaired as compared to single digits. However, two-digit numerals were only studied in the context of a single task (reading aloud), and one may wonder whether they could be identified correctly in other contexts. We examined if the dissociation between reading aloud and comparison, already observed in several patients with large posterior left-hemispheric lesions (Cohen + and Dehaene, 1991; Dehaene + and Cohen, 1991; Cohen + et al., 1994), could be replicated in pure alexic patients.

A list of 22 pairs of two-digit arabic numerals was constituted. The larger number was on the right in half the pairs and on the left in the other half. A second list of 22 pairs was derived from the original list by simply inverting the right and left numerals in each pair. In the comparison condition, the patients were presented with both lists, and asked to point to the larger number of each pair. They were instructed to refrain from reading the stimuli aloud. In the reading condition, patient G.O.D. was presented with the original list and patient S.M.A. with both lists, one pair at a time, and asked to read aloud the two numerals. For instance, the patients were expected to respond 'forty seven, fifty two' when presented with the pair 4752 .

As shown in Fig. 8 and Table 3, the comparison task was extremely easy for both patients and did not yield a single error. In the reading task, by contrast, patient G.O.D. made at least one 
error on $90.9 \%$ of trials and S.M.A. on $88.6 \%$ of trials. These errors affected 31 out of 44 (70.4\%) numbers in G.O.D., and 61 out of 88 numbers in S.M.A. (69.3\%). A high rate of perseverations was observed. Both patients tended to repeat over and over again the same numbers regardless of the actual stimulus. For instance when reading the sequence of pairs 68 71, 39 42, 3229 and 26 62, patient G.O.D. said '68 796979794969 69'.

The above difference in overall performance level between comparison and reading aloud, although very suggestive in itself, does not strictly demonstrate that stimuli were better identified in the former than in the latter task. More critical is the frequency of those reading errors that did not respect the relative magnitude of the two stimuli (for instance, patient G.O.D. read 7876 as 'seventy eight, seventy nine'). If reading aloud rests on a less effective number identification procedure than comparison, this particular kind of reading error could be more frequent than comparison errors. In contrast, if the same identification procedure intervenes in both tasks, there should be no such difference. In the reading task, patient G.O.D. made $22.7 \%$ errors that did not respect the relative magnitude of the stimuli, while patient S.M.A. made $38.6 \%$ errors of this kind (see Table 3 ). These error rates were significantly higher than the (null) error rates observed in the comparison task (G.O.D.: $x^{2}(1)$ $=7.82, P=0.0052$, Yates' correction applied; S.M.A.: $\left.X^{2}(1)=21.07, P=0.000004\right)$.

In brief, this experiment provided perhaps the clearest evidence of contrasted identification abilities across tasks. Both patients performed very poorly when reading aloud two-digit numerals (as was already shown in previous sections), but they were perfectly accurate in comparing two such numerals. These results suggest that two-digit numerals, like single digits, may be identified by two different systems, only one of which is impaired in pure alexia.

\section{Reading spelled-out numerals}

The data described so far concerned only arabic numerals. As a final point, we investigated whether similar effects would obtain with alphabetically spelled-out numerals, using the same tasks as with arabic numerals.

The patients were presented with pairs of spelled-out numerals, printed one word above the other. Stimuli were a translation of the list of digit pairs used in previous tasks. Four tasks were used. In the simple reading condition, the patients were asked to read aloud each word. In the reading-and-addition condition, they were asked to read both words aloud, and then to produce their sum. In the reading-and-comparison condition, the patients had to read both words aloud and then to name the larger one and point to it. For instance, on presentation of the pair FIVE/THREE, the patients were expected to respond 'five, three ... eight' in the reading-and-addition task, and 'five, three ... five' in the reading-and-comparison task. Finally, in the silent comparison task, they simply had to point to the larger number without reading it aloud. In each condition, patient G.O.D. received the experimental list twice, and patient S.M.A. once.

As shown in Table 4, and contrary to what was observed with arabic digits, task structure did not exert any influence on the naming of number words. The rate of naming errors was not different across the three conditions in which overt reading was required (G.O.D.: $x^{2}(2)=$ 1.64, $P=0.44$; S.M.A.: $x^{2}(2)=0.55, P=0.76$ ). In the reading-and-comparison and the reading-and-addition tasks, the patients' final answer was always in keeping with their naming of the operands. In the reading-and-addition task, they always produced the correct sum of the misread operands (except for one omission in patient G.O.D.). In the reading-and-comparison 
task, they always chose the larger number of the two that they had produced (for instance, when presented with the pair 'two three', patient G.O.D. said 'six, three, it's six').

In the silent comparison task, patients G.O.D. and S.M.A. made $12.5 \%$ and $15.0 \%$ errors, respectively. There was no difference in the rate of comparison errors between the silent comparison and the reading-and-comparison tasks.

View this table: Table 4. Error rates (\%) with alphabetically spelled-out numerals [in this window]

[in a new window]

Pooling the naming data from the simple reading, the reading-and-addition and the readingand-comparison tasks, patient G.O.D. made $19.6 \%$ errors, and patient S.M.A. $10.0 \%$ errors. It may seem that both patients performed rather well considering the severity of their pure alexia. However, this relatively good performance can probably be attributed to a strategy of reading only the first few letters of each word. Such a strategy can be highly successful with spelled-out numerals because the set of possible stimuli is small and because the French numerals from one to nine start with a different letter, except for 'six' and 'sept'. When the set of stimuli was extended to all 23 single-word numerals, both patients made significantly more reading errors (G.O.D.: 20 errors out of 46 [43.5\%], $X^{2}(1)=12.3, P=0.00046$; S.M.A.: 18 errors out of 46 [39.1\%], $\left.x^{2}(1)=19.1, P=0.000013\right)$. Many of these errors consisted of substitutions of words starting with the same letter (for instance, patient G.O.D. read 'quatre' [4] as 'quinze' [15] and 'trente' [30] as 'treize' [13]).

In conclusion, with spelled-out numerals, the absence of any performance variation across tasks suggested that the same word identification procedure operated in all conditions. This contrasted sharply with the reading of arabic numerals, which showed a consistent influence of task structure. 


\section{Summary of results}

We have explored the number processing abilities of two patients suffering from typical pure alexia. In most experiments, we used identical stimuli and examined the variations in performance as a function of task demands. We first showed that the patients were better at reading two digits as a pair than as a two-digit numeral. It appeared next that the patients performed perfectly when comparing two digits, but quite poorly when adding them, even though addition of spoken numerals was perfect. When asked to read aloud the operands while performing the addition and comparison tasks, the patients made many more reading errors during addition than during comparison. This suggested that their poor addition performance was due to a misidentification of arabic digits. This finding was extended to an addition verification task, in which addition and comparison processes were combined on each trial. A similar dissociation between excellent comparison and impaired reading aloud was documented with two-digit numerals. Finally, the variation of performance with task demands was shown not to prevail with spelled-out numerals.

\section{Models of number processing}

We first address the question of how our observations on number reading and calculation fit with available models of number processing. A currently influential model has been proposed by McCloskey and his colleagues (McCloskey + et al., 1985; McCloskey*, 1992). This model assumes that when subjects are required to process arabic numerals, an arabic comprehension module comes into play which converts the input digit string into an amodal semantic representation of the number.

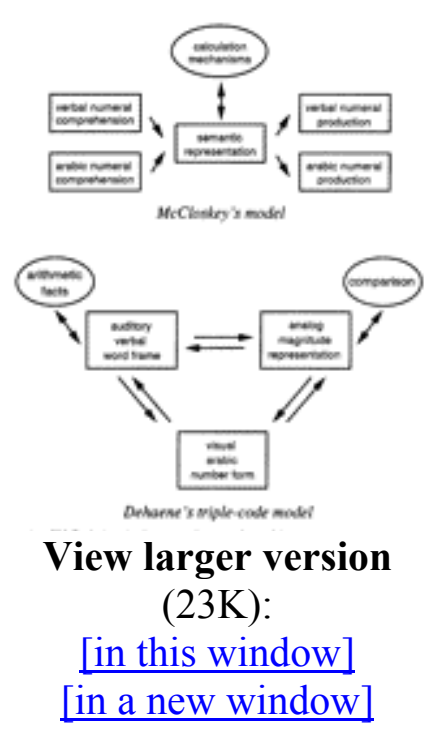

Fig. 9 Schematic representation of McCloskey's and Dehaene's number processing models.

The same semantic representation can also be evoked, via other specialized comprehension modules, by stimuli in other notations such as spelled-out numerals or spoken number words. All subsequent processes, such as addition, comparison or reading aloud, are assumed to operate exclusively on this central abstract representation (see Fig. 9). 
The present observations appear incompatible with McCloskey's framework. Our patients made no errors in comparing arabic numerals, which in McCloskey's model implies that the arabic comprehension module and the comparison procedure were preserved. Likewise both patients were perfect in producing orally the results of additions that were read aloud to them. This implies that the addition process itself, the spoken comprehension, and the spoken production modules, were intact. Finally, writing arabic numerals to dictation was perfect, indicating that the written production module was intact. One should therefore reach the paradoxical conclusion that the entire number processing system depicted in Fig. 9 was intact! As a matter of fact, however, addition and reading aloud of arabic numerals were severely affected. Our results demonstrate that the performance in identifying arabic numbers varies depending on the task, which is fundamentally at odds with McCloskey's hypothesis of a taskindependent comprehension module.

More generally, our results appear difficult to reconcile with the hypothesis of a partial deficit affecting a single visual identification system (Bub + et al., 1989; Farah + and Wallace, 1991; Friedman + et al., 1993). Several results seem difficult to account for in such a framework. First, it is not clear why a partially disrupted visual number form should be sufficient to compare two arabic numerals or to name single digits, but not to perform mental arithmetic or to read aloud complex numerals. Both patients were essentially perfect in comparing arabic numerals differing by as little as one unit, a situation which prevailed in one-quarter of the digit comparison trials. Even though magnitude comparison may be considered as a kind of category judgement, it seems unlikely that it can be performed with such accuracy on the basis of a partially impaired identification system that would generate numerous naming and calculation errors, sometimes on the very same trial. Second, the hypothesis of a partial deficit does not account for the performance variation in reading aloud, depending on the task in which patients were engaged. As an illustration of this point, remember that, in the addition verification task, patient G.O.D. made more than $40 \%$ errors when reading the operand digits, but not a single error when reading the proposed sum.

An alternative model of number processing has been put forward by Dehaene * (1992; Cohen et al., 1994). This triple-code model supposes that number processing is subserved by three distinct mental representations of numbers: a visual arabic code (the visual number form), an auditory verbal code, and a semantic magnitude code (see Fig. 9). Dedicated transcoding routines allow for a conversion between any two of these codes. Finally each elementary computation is assumed to operate over specified input and output codes. In particular, data from normal subjects suggested that number comparison operates only on the analogue magnitude representation, whereas arithmetic fact retrieval relies on the verbal code. 


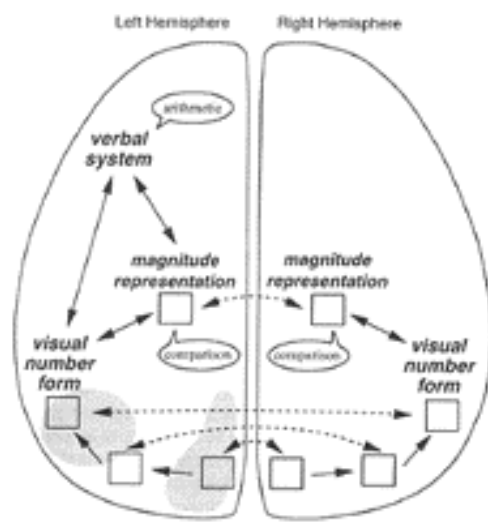

View larger version $(52 \mathrm{~K})$ : [in this window] [in a new window]
Fig. 10 Model of number processing combining the main features of the anatomo-functional model of reading displayed in Fig. 1 and of the triple-code theory displayed in Fig. 9.

A somewhat similar framework has been proposed by Campbell and Clark under the name of encoding complex theory (Campbell + and Clark, 1988; Clark $\star$ and Campbell, 1991).

It is possible to 'lesion' the triple code model in such a way as to account naturally for the main features of the present data. The deficit in reading arabic numerals can be attributed to a single lesion affecting the direct transcoding route linking the visual arabic code to the auditory verbal code. Such a lesion leaves intact the translation of arabic numerals to the magnitude code, thus explaining the excellent performance of patients in number comparison tasks. The sparing of the auditory verbal representation and its attached computations explains that all tasks involving only spoken numerals could be performed adequately.

\section{Anatomical implementation}

The triple code model was initially developed as a purely psychological construct. However, since it came to be used as a framework for the interpretation of neuropsychological deficits, it seems desirable to specify as well as possible the cerebral implementation of its components (Dehaene+ and Cohen, 1995).

There is clear evidence from commissurotomized patients that either hemisphere is able to identify visually presented arabic digits, as well as to decide accurately which of two digits is larger (Sergent*, 1990; Seymour $*$ et al., 1994). These observations suggest that the visual arabic code, as well as the magnitude code, are represented in both hemispheres in a duplicate form. In contrast, an isolated right hemisphere is unable to name digits aloud, and to perform even simple arithmetic calculations, while these two tasks are easy for an isolated left hemisphere (Gazzaniga + and Hillyard, 1971; Gazzaniga + and Smylie, 1984). In the context of the triple code model, verbal output and arithmetic computations are dependent on the auditory verbal code, which therefore appears to be restricted to the left hemisphere. This pattern of hemispheric specialization is further supported by the observation that patients with extensive left hemispheric lesions, while unable to solve any arithmetic problem, may none the less compare accurately one- or even two-digit numerals (Grafman + et al., 1989; Dehaene+ and Cohen, 1991). Thus, the triple-code model reduces to the large-scale cerebral network depicted on Fig. 10 (see Dehaene+ and Cohen, 1995, for further discussion).

Within this model, a left infero-mesial occipito-temporal lesion, as observed in the two patients that we have described, would disrupt the left hemispheric visual number form itself, 
its afferents, or both. This would result precisely in the functional impairment proposed in the previous section. First, reading aloud arabic numerals should be impaired, due to an inability to transmit digit identities directly from the visual system to the verbal system. However, partial reading may in principle remain possible through an indirect reading pathway involving the right hemisphere. As apparent on Fig. 10, numerals can be identified by the right hemispheric visual system, and then translated into a semantic representation specifying, in particular, the associated magnitude. This magnitude representation can be transferred to the left hemisphere, and eventually activate the verbal system, allowing for the production of a verbal response. Second, calculation impairments are also predicted. Assuming that mental arithmetic relies on verbal abilities, it follows that it should also be dependent on direct input from the left visual system (Dehaene*, 1992). Accordingly, the patients made numerous addition errors resulting from a faulty identification of the operands. Finally, and in contrast with addition, number comparison should be preserved because the intact right hemisphere, by itself, possesses sufficient identification and magnitude processing abilities.

\section{Further details}

Although this framework appears to account naturally for the behaviour of the patients we have described, several aspects of their reading performance, particularly relating to the influence of task structure, deserve further discussion.

First, why do patients with pure alexia generally have difficulties reading aloud multidigit numerals, but not single digits? We see at least three explanations that are not mutually exclusive and that emphasize either the visual, the semantic, or the verbal differences between single digits and multidigit numerals. At the visual level, the intact right visual system is able to recognize simple symbols (which may be viewed as essentially equivalent to single objects), but it might be unable to compute a structural representation for objects comprising multiple parts, such as words or digit strings (Farah $\star$, 1990). At the semantic level, each small number probably evokes specific semantic associations, including a precise magnitude estimate, which, when transmitted to the left hemisphere, may fully specify the adequate verbal response. Most large numbers, however, do not receive such a specific semantic interpretation. There may be exceptions, such as round numbers, famous dates, or brands of cars, and indeed we have observed a relative sparing of such numerals in a patient with deep dyslexia, whose reading was also presumed to rely on a right-hemispheric semantic route (Cohen $*$ et al., 1994). Finally, at the verbal level, reading aloud multidigit numerals requires assembling an elaborate sequence of words obeying language-specific rules. This is not unlike the phonological assembly required for naming non-words, and may demand specific visual number form information for input, rather than semantic information. By contrast, naming single digits only requires retrieving a single word, a process analogous to the naming of ordinary objects and which may be performed on the basis of semantic information provided by the right hemisphere.

Secondly, why are single digits better read than single letters (Dejerine $\star$, 1892; Holender $\star$ and Peereman, 1987)? Again, several explanations may be proposed. At the visual level, digits and letters are equally simple visual objects, but letters are more numerous and may be more similar to one another. A similar argument can be made at the verbal level since letter names often sound very similar (Alajouanine + et al., 1960). Finally, at the semantic level, contrary to digits, letters do not possess much intrinsic meaning that could help specify unambiguously their identity to the left hemisphere (Geschwind $*$, 1965a; Holender $*$ and Peereman, 1987). Whatever is the correct explanation, it is noteworthy that a similar advantage of digits over letters has been observed with stimuli flashed to the isolated right hemisphere of six split-brain 
patients (Teng + and Sperry, 1973). This observation is in good agreement with the idea that the discrepancy between digits and letters observed in pure alexics reflects a contribution of the right hemisphere.

Thirdly, how can we explain the remarkable variation in reading aloud even single digits, depending on the task context? Our two patients made almost no errors when reading digit pairs in the context of a comparison task, whereas many reading errors occurred in the context of an addition task. Why were they unable to use their right-hemispheric reading route equally well in both tasks? Only tentative solutions can be offered for this observation. We speculate that when subjects engage in a given cognitive task, a set of appropriate cerebral circuits is selected. There is good evidence from functional brain imagery in normal subjects that changes in task structure result in major reorganizations of active brain areas, even when stimuli are kept identical (e.g. Corbetta + et al., 1990; Posner + and Raichle, 1994). In the particular case of number processing, we have observed significant differences in regional blood flow during multiplication or comparison of the same pairs of digits (Dehaene + et al., in preparation). Such task-related reorganization may affect reading processes. The cortical network for solving addition problems presumably involves only left-hemispheric structures, including the lesioned visual number form system. Thus the patients are not induced towards an optimal use of their intact right-hemispheric reading pathway. Conversely, number comparison can be performed by either hemisphere, and electroencephalographic evidence indicates that it recruits bilateral posterior areas for digit identification and magnitude processing (Dehaene*, 1995). In this situation, attentional resources are allocated to the right hemisphere, which can therefore contribute effectively to the reading process. Moreover, in the course of the comparison process, the right hemisphere is obliged to characterize as precisely as possible the magnitude of the target digits. It is therefore not surprising that this refined analysis may increase the accuracy of reading aloud.

A related hypothesis has been put forward by Landis $*$ et al. (1980; Regard + et al., 1994) and by Cipolotti + (1995). They suggest that right-hemispheric reading abilities are inhibited when the left-hemispheric linguistic system is actively functioning. According to this hypothesis, when subjects are engaged in an addition task, the right-hemispheric reading route would be repressed.

Finally, why was there no influence of task demands on the naming of spelled-out numerals? Our personal observations of a patient with a posterior callosal lesion suggest that the right hemisphere may not be able to recognize written number words, although we know of no published data on this topic. In agreement with this hypothesis, the two patients reported here seemed to rely on the identification of the first few letters in order to read aloud spelled-out numerals. Because this was their only available procedure for reading number words, we speculate that the patients were obliged to use it irrespective of the subsequent task. In general, number words seem to behave like abstract, infrequent, or grammatical words which the right hemisphere is unable to identify. With more concrete and imageable words, task effects on word identification can be observed, as reviewed in the introduction (Coslett + et al., 1993).

\section{Related findings in number processing}

Previous reports have already mentioned in passing that the identification of arabic numerals is generally preserved in pure alexics, and that performance may vary with task demands. Dejerine + (1892) noted that 'the patient recognizes very well all digits', while 'he cannot recognize a single letter'. Dejerine also observed that multidigit numerals seemed not to enjoy the same advantage. In order to read aloud the numeral 112, the patient first had to name each 
digit in turn. Although most subsequent studies of pure alexia did not specifically consider the case of arabic numerals, a review of the relevant literature indicates that this pattern of behaviour seems to be the rule (Holender $*$ and Peereman, 1987). For instance, the patient described by Kreindler $*$ and Ionasescu (1961) could read aloud single digits, but produced many substitution errors similar to those of patients G.O.D. and S.M.A. when he was asked to name multidigit numerals. Moreover, there were some suggestions that this patient showed a dissociation between addition and comparison tasks reminiscent of the present observations.

More recently, McNeil $\star$ and Warrington (1994) have described a patient who shared several striking features with the present cases. Although he suffered from a bilateral brain tumour and also had nominal aphasia, the patient mainly showed severe alexia without agraphia. Just like patients G.O.D. and S.M.A., he could read single digits much better than digits embedded in complex arabic numerals, and his reading errors were all digit substitutions. Furthermore, he was severely impaired in adding or multiplying written numerals, while he was excellent in comparing them. Most importantly, he was excellent in solving the very same arithmetic problems on oral presentation, suggesting that the identification of written digits operated differently during addition and comparison.

\section{Extension to pure alexia and optic aphasia}

Outside of the numerical domain, an influence of task structure on the identification of visual stimuli has been evidenced with various types of material other than written numerals, including words and even objects or pictures. As reviewed in the introduction, patients with pure alexia may display residual word identification abilities in tasks such as lexical decision or semantic classification (Coslett $*$ et al., 1993). Moreover, patients with the syndrome of optic aphasia are able to access specific information about objects or colours that they cannot name or even describe on visual presentation (Freund $\star$, 1889; Lhermitte $\star$ and Beauvois, 1973; Coslett* and Saffran, 1989).

All such patients may be characterized by a selective naming deficit on visual presentation, with evidence from non-verbal tasks that the stimuli were properly recognized. Another common characteristic is the frequent occurrence of perseverations in naming. The patients reported here often produced the same response for many trials in a row when naming letters, words or arabic numerals. Similar perseverative behaviour has been reported in patients with optic aphasia (Lhermitte $\bullet$ and Beauvois, 1973; Farah $\bullet$, 1990).

A homogeneous framework can be proposed to account for these common characteristics of pure alexia (for words and for arabic numerals) and optic aphasia. First, the left-hemispheric verbal system does not receive normal inputs from regions of the visual system specialized for word, digit, object or colour recognition, and thus naming of such stimuli is affected.

Perseverations may be considered as 'attempts to fill gaps in the information available to [the] speech area' (Geschwind + 1965b, p. 590) when the verbal system is deprived of adequate inputs. This typically results from a lesion of left-hemispheric visual areas associated with a posterior interhemispheric disconnection. Second, the intact right-hemispheric visual system may remain able to identify and categorize some visual stimuli, allowing patients to show a variety of residual abilities.

This interpretation is uncontroversial in the case of split-brain patients who fail to name stimuli presented in their left visual hemifield. In split-brains, because of the complete disconnection, stimuli in the left hemifield cannot gain access to the verbal system, resulting in left alexia and visual anomia with frequent perserverative errors. At the same time, accurate 
identification can be demonstrated when the patients are required to respond using their right hemisphere only (e.g. Gazzaniga , 1970).

A similar interpretation may apply in the case of pure alexia and optic aphasia. It is, however, made more complex by the fact that the interhemispheric disconnection is only very partial and that therefore the two hemispheres can still exchange a substantial amount of information. In particular it seems likely that the right hemisphere can extract and transfer to the left verbal system some semantic information about the stimuli. Such partial transfer has been proposed as the basis for the residual reading abilities and semantic errors in patients with deep dyslexia or pure alexia (Coltheart*, 1980; Shallice*, 1988; Cohen $*$ et al., 1994). In general, performance in any visuo-verbal task will therefore depend, first, on the competence of the isolated right hemisphere, and secondly, on whether sufficient information regarding the stimulus can be received and exploited by the left hemisphere.

\section{Acknowledgments}

The authors thank Professors P. Brunet and B. Dubois for referring the patients. This work was supported in party by the Institut National de la Santé et de la Recherche Médicale (INSERM) and the Faculté de Médecine Pitié-Salpêtrière.

\section{Notes}

${ }^{1}$ Service de Neurologie, Clinique Paul Castaigne, Hôpital de la Salpêtrière, 47 Boulevard de l'Hôpital, 75651 Paris Cedex 13,

${ }^{2}$ Laboratoire de Sciences Cognitives et Psycholinguistique EHESS, INSERM and CNRS, Paris, France,

\section{REFERENCES}


Alajouanine T, Lhermitte F, de Ribaucourt-Ducarne B. Les alexies agnosiques et aphasiques. In: Alajouanine T, editor. Les grandes activités du lobe occipital. Paris: Masson, 1960: 23560 .

Binder JR, Mohr JP. The topography of callosal reading pathways: A case control analysis. Brain 1992; 115: 1807-26.

Bub DN, Black S, Howell J. Word recognition and orthographic context effects in a letter-byletter reader. Brain and Language 1989; 36: 357-76.

Campbell JID, Clark JM. An encoding-complex view of cognitive number processing: Comment on McCloskey, Sokol, \& Goodman (1986). Journal of Experimental-Psychology: General 1988; 117: 204-14.

Cipolotti L. Multiple routes for reading words, why not numbers? Evidence from a case of arabic numeral dyslexia. Cognitive Neuropsychology 1995; in press.

Clark JM, Campbell JID. Integrated versus modular theories of number skills and acalculia. Brain and Cognition 1991; 17: 204-39.

Cohen L, Dehaene S. Neglect dyslexia for numbers? A case report. Cognitive Neuropsychology 1991, 8: 39-58.

Cohen L, Dehaene S, Verstichel P. Number words and number non-words: a case of deep dyslexia extending to arabic numerals. Brain 1994; 117: 267-79.

Coltheart M. Deep dyslexia: a right-hemisphere hypothesis. In: Coltheart M, Patterson KE, Marshall JC, editors, Deep dyslexia. London: Routledge, 1980: 326-80.

Corbetta M, Miedzin FM, Dobmeyer S, Shulman GL, Petersen SE. Attentional modulation of neural processing for shape, color, and velocity in humans. Science 1990; 248: 1556-9.

Coslett HB, Monsul N. Reading with the right hemisphere: evidence from transcranial magnetic stimulation. Brain and Language 1994; 46: 198-211.

Coslett HB, Saffran EM. Preserved object recognition and reading comprehension in optic aphasia. Brain 1989; 112: 1091-110.

Coslett HB, Saffran EM, Greenbaum S, Schwartz H. Reading in pure alexia: the effect of strategy. Brain 1993; 116: 21-37.

Damasio H, Damasio AR. Lesion analysis in neuropsychology. Oxford: Oxford University Press, 1989.

Damasio AR, Damasio H. Anatomical basis of pure alexia. Neurology 1983; 33: 1573-83.

Dehaene S. Varieties of numerical abilities. Cognition 1992; 44: 1-42. 
Dehaene S. The organization of brain activations in number comparison: event-related potentials and the additive-factors method. Journal of Cognitive Neuroscience 1995; in press.

Dehaene S, Cohen L. Two mental calculation systems: a case study of severe acalculia with preserved approximation. Neuropsychologia 1991; 29: 1045-74.

Dehaene S, Cohen L. Towards an anatomical and functional model of number processing. Mathematical Cognition 1995; in press.

Dehaene S, Dupoux E, Mehler J. Is numerical comparison digital? Analogical and symbolic effects in two-digit number comparison. Journal of Experimental Psychology: Human Perception and Performance 1990; 16: 626-41.

Dejerine J. Contribution à l'étude anatomo-pathologique et clinique des différentes variétés de cécité verbale. Comptes Rendus des Seances de la Societé de Biologie 1892; 4: 61-90.

Eviatar Z, Menn L, Zaidel E. Concreteness: nouns, verbs and hemispheres. Cortex 1990; 26: $611-24$

Farah MJ. Visual agnosia: disorders of object vision and what they tell us about normal vision. Cambridge, Massachusetts: MIT Press, 1990.

Farah MJ, Wallace MA. Pure alexia as a visual impairment: a reconsideration. Cognitive Neuropsychology 1991; 8: 313-34.

Freund CS. Über optische Aphasie und Seelenblindheit. Archiv für Psychiatrie und Nervenkrankheit 1889; 20: 276-97.

Friedman RB, Alexander MP. Pictures, images, and pure alexia: a case study. Cognitive Neuropsychology 1984; 1: 9-23.

Friedman RB, Beeman M, Lott SN, Link K, Grafman J, Robinson F. Modality-specific phonological alexia. Cognitive Neuropsychology 1993; 10: 549-68.

Gazzaniga M. The bissected brain. New York: Appleton Century Crofts, 1970.

Gazzaniga MS, Hillyard SA. Language and speech capacity of the right hemisphere. Neuropsychologia 1971; 9: 273-80.

Gazzaniga MS, Smylie CS. Dissociation of language and cognition: a psychological profile of two disconnected right hemispheres. Brain 1984; 107: 145-53.

Geschwind N. Disconnexion syndromes in animals and man: Part I. Brain 1965a; 88: 237-94.

Geschwind N. Disconnexion syndromes in animals and man: Part II. Brain 1965b; 88: 585644.

Goodglass H, Kaplan E. The Boston Diagnostic Aphasia Examination. Philadelphia: Lea \& Febiger, 1972. 
Grafman J, Kampen D, Rosenberg J, Salazar A, Boller F. Calculation abilities in a patient with a virtual left hemispherectomy. Behavioral Neurology 1989; 2: 183-94.

Grober E, Buschke H, Crystal MD, Bang MA, Dresner R. Screening for dementia by memory testing. Neurology 1988; 38: 900-3.

Gr OJ, Landis T. Lost letters: pure alexia. In: Visual agnosias and other disturbances of visual perception and cognition. London: Macmillan Press, 1991: 333-58.

Hines D. Differences in tachistoscopic recognition between abstract and concrete words as a function of visual half-field and frequency. Cortex 1977; 13: 66-73.

Holender D, Peereman R. Differential processing of phonographic and logographic singledigit numbers by the two hemispheres. In: Deloche G, Seron X, editors, Mathematical disabilities: a cognitive neuropsychological perspective. Hillsdale, New Jersey: Lawrence Erlbaum Associates, 1987: 43-85.

Kreindler A, Ionasescu V. A case of 'pure' word blindness. Journal of Neurological and Neurosurgical Psychiatry 1961; 24: 275-80.

Landis T, Regard M, Serrat A. Iconic reading in a case of alexia without agraphia caused by a brain tumor: A tachistoscopic study. Brain and Language 1980; 11: 45-53.

Lhermitte F, Beauvois MF. A visual-speech disconnexion syndrome: report of a case with optic aphasia, agnosic alexia and colour agnosia. Brain 1973; 96: 695-714.

Mazaux JM, Orgogozo JM. Echelle d'évaluation de l'aphasie: adaptation française du Boston Diagnostic Aphasia Examination. Issy-les-Moulineaux: Editions Scientifiques et Psychologiques, 1982.

McCloskey M. Cognitive mechanisms in numerical processing: evidence from acquired dyscalculia. Cognition 1992; 44: 107-57.

McCloskey M, Caramazza A, Basili A. Cognitive mechanisms in number processing and calculation: evidence from dyscalculia. Brain and Cognition 1985; 4: 171-96.

McCloskey M, Sokol SM, Goodman-Schulman RA, Caramazza A. Cognitive representations and processes in number production: evidence from cases of acquired dyscalculia. In:

Caramazza A, editor, Advances in cognitive neuropsychology and neurolinguistics. Hillsdale, New Jersey: Lawrence Erlbaum Associates, 1990: 1-32.

McNeil JE, Warrington EK. A dissociation between addition and subtraction with written calculation. Neuropsychologia 1994; 32: 717-28.

Patterson K, Vargha-Khadem F, Polkey CE. Reading with one hemisphere. Brain 1989; 112 : $39-63$.

Petersen SE, Fox PT, Snyder AZ, Raichle ME. Activation of extrastriate and frontal cortical areas by visual words and word-like stimuli. Science 1990; 249: 1041-4. 
Posner MI, Raichle ME. Images of mind. New York: Scientific American Library, 1994.

Regard M, Cook ND, Wieser HG, Landis T. The dynamics of cerebral dominance during unilateral limbic seizures. Brain 1994; 117: 91-104.

Reuter PA, Brunn JL. A prelexical basis for letter-by-letter reading. Cognitive Neuropsychology 1990; 7: 1-20.

Sergent J. Furtive incursions into bicameral minds: integrative and coordinating role of subcortical structures. Brain 1990; 113: 537-68.

Seymour SE, Reuter-Lorenz PA, Gazzaniga MS. The disconnection syndrome: basic findings reaffirmed. Brain 1994; 117: 105-15.

Shallice T. From neuropsychology to mental structure. Cambridge: Cambridge University Press, 1988.

Shallice T, Saffran EM. Lexical processing in the absence of explicit word identification: Evidence from a letter-by-letter reader. Cognitive Neuropsychology 1986; 3: 429-58.

Snodgrass JG, Vanderwart M. A standardized set of 260 pictures: norms for name agreement, image agreement, familiarity, and visual complexity. Journal of Experimental Psychology: Human Learning 1980; 6: 174-215.

Teng EL, Sperry RW. Interhemispheric interaction during simultaneous bilateral presentation of letters or digits in commissurotomized patients. Neuropsychologia 1973; 11: 131-40.

Warrington EK, Shallice T. Word-form dyslexia. Brain 1980; 103: 99-112.

Zaidel E, Peters AM. Phonological encoding and ideographic reading by the disconnected right hemisphere: two case studies. Brain and Language 1981; 14: 205-34.

Received on 1 February 1995; resubmitted on 15 May 1995; accepted on 16 May 1995

\section{This article has been cited by other articles:}

- Leff, A. P., Crewes, H., Plant, G. T., Scott, S. K., Kennard, C., Wise, R. J. S. (2001). The functional anatomy of single-word reading in patients with hemianopic and pure alexia. Brain 124: 510-521 [Abstract] [Full Text]

- Chochn, F., Cohen, L., Moortele, P. F. v. d., Dehaene, S. (1999). Differential Contributions of the Left and Right Inferior Parietal Lobules to Number Processing. J. Cogn. Neurosci. 11: 617-630 [Abstract] [Full Text] 
- Cohen, L., Dehaene, S., Naccache, L., Lehericy, S., Dehaene-Lambertz, G., Henaff, M.-A., Michel, F. (2000). The visual word form area: Spatial and temporal characterization of an initial stage of reading in normal subjects and posterior splitbrain patients. Brain 123: 291-307 [Abstract] [Full Text] 